\title{
Editorial, Theranostics.
}

\section{Theranostics in Prostate Cancer.}

Zidan, $\mathbf{L}^{1}$.

${ }^{2}$ Nuclear medicine Unit, Faculty of Medicine, Cairo University.

\section{ABSTRACT:}

Metastatic castration resistant prostate cancer (mCRPC) is a highly mortal disease, requiring safe and effective therapeutic agents to improve the patient outcome. Prostate-specific membrane antigen (PSMA) is over expressed in many prostate cancer cells, and act as target for molecular imaging (MI) and radionuclide therapy (RNT). The introduction of Gallium-68 (68Ga) PSMA- PET/CT in 2012 has changed the management of prostate cancer. In addition to its importance in identifying PSMA expression for selection of patients suitable for PSMA RNT, PSMA- PET/CT showed superior diagnostic accuracy to combined CT and bone scan. Lutetium-177 (177Lu) PSMA-617 is the most investigated PSMA radio ligand for RNT in prostate cancer, it is a radiolabelled small molecule, binds with high affinity to PSMA, enabling beta-particles emissions with short tissue penetration depth (mean range of $0.7 \mathrm{~mm}$ and maximum range of $2.1 \mathrm{~mm}$ in soft tissue). Since 2014 multiple studies showed a highly impressive therapeutic efficacy and safety of ${ }^{177}$ Lu-PSMA RNT. The evidence achieved by these studies paved the way to ongoing randomized clinical trials, and currently this therapy is being applied and clinically accepted in many centers worldwide. The aim of this article is to share the practical aspect of PSMA Theranostics for prostate cancer, including patient selection, therapy protocol and follow-up. 


\section{INTRODUCTION:}

Prostate cancer is the second most common cancer among men and one of the leading cause of cancer death, after lung cancer ${ }^{(\mathbf{1})}$.

Its biological behavior differs according to the disease stage. Although localized disease may be cured with surgery or radiotherapy, distant metastatic disease may develop in many patients. Androgen deprivation therapy (ADT) with or without docetaxel is recommended as a first-line treatment of distant metastatic prostate cancer ${ }^{(2)}$, however eventually most of the patients progress and develop metastatic castration resistant prostate cancer (mCRPC). Enzalutamide and Abiraterone, are antiandrogen agents that have been approved for mCRPC; However, they carry serious side effects, such as cardiac morbidity ${ }^{(2)}$. Other treatment options of mCRPC include; cabazitaxel, sipuleucel-T, and in bone metastases, Radium-223 dichloride ${ }^{(3)}$.

Prostatic specific membrane antigen (PSMA), is a type II transmembrane glycoprotein, that is highly expressed in most prostate cancer, it act as a target for molecular imaging (MI) and radionuclide therapy (RNT) in the sense of a theranostic approach (4). PSMA PET/CT provide higher diagnostic accuracy than conventional imaging in prostate cancer ${ }^{(5)}$, in addition to its importance to assess PSMA expression for selection of patients for PSMA
RNT (6-8). Although ${ }^{68}$ Ga-PSMA PET/CT has been used in the majority of the studies, other ligands such as ${ }^{18} \mathrm{~F}-\mathrm{DCFBC},{ }_{18} \mathrm{~F}$ DCFPyL and 18F-PSMA-1007 have emerged for PSMA PET/CT imaging ${ }^{(9-11)}$.

PSMA theronostic with PSMA- small molecules or targeted antibodies, conjugated to either alpha or beta-particle emitting radioisotopes, have shown highly promising therapeutic efficacy in prostate cancer. PSMA-targeted small molecules have the advantage of the rapid internalization and blood pool clearance, compared to PSMAtargeted antibody ${ }^{(\mathbf{1 2})}$.

Although alpha-particle emitters have higher energy than beta-particle emitters, they show non-specific binding to normal tissue and carry more toxicity to salivary gland and kidneys ${ }^{(13)}$. The therapeutic radionuclide of choice is the beta-particle emitters, Lutetium$177\left({ }^{177} \mathrm{Lu}\right)$; it has a short tissue penetration depth and lower abundance of gamma emissions.

${ }^{177}$ Lu-DOTA-PSMA-617 (177Lu-PSMA-617) and ${ }^{177} \mathrm{Lu}$-DOTAGA-PSMA-I\&T $\quad(177 \mathrm{Lu}-$ PSMA-I\&T) are the most commonly used PSMA-targeted small molecule-based RNT (6). Several studies using 177Lu-PSMA-617 in Europe and Australia, showed significant results with decrease in PSA and minimal toxicity ${ }^{(7,8,14-18)}$. 
Currently there are randomized clinical trials on the efficacy of PSMA RNL, including; the VISION trial, NCT03511664 (it's an international, prospective multicentre, randomized phase 3 trial opened in May 2018) and TheraP trial, NCT03392428 (it's a multicentre randomized trial in Australia) ${ }^{(19}$, 20).

\section{Patient Selection:}

A multidisciplinary uro-oncology team, including a medical oncologist, radiation oncologist, and urology surgeon as well as nuclear medicine physician is recommended for selection of prostate cancer patients suitable for PSMA RNT. Castration resistant prostate cancer with progressive disease and rising PSA level as recommended by the Prostate Cancer Trials Working Group 3 (PCWG 3) criteria, in addition adequate bone marrow, renal and liver function are prerequisites ${ }^{(21)}$. Presence of sufficient PSMA expression remains the gatekeeper for patient selection, while the threshold of PSMA uptake on PSMA PET/CT below which PSMA RNT is less likely to be effective is still not clear. Eligibility of PSMA RNT in the VISION trial required $68 \mathrm{Ga}-\mathrm{PSMA}-11$ uptake above normal liver activity, the TheraP trial required certain SUV threshold (SUV max $\geq$ 20 at a site of disease and SUV $\max >10$ at sites of measurable disease ( $\geq 10 \mathrm{~mm})$, and $\mathrm{Lu}$ PSMA trial mandated ${ }^{68}$ Ga-PSMA-11 uptake

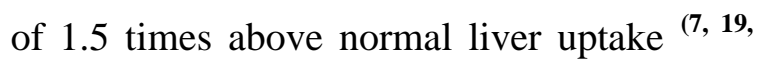
20). There is evidence of cellular and molecular heterogeneity in prostate cancer, and this may explain the non-uniform therapeutic response. The importance of dual tracer MI has been highlighted in Lu PSMA study, where all patients underwent ${ }_{68} \mathrm{Ga}-$ PSMA-11 and ${ }^{18}$ F-FDG PET/CT for screening, $11 \%$ of the screened patient were excluded due to presence of FDG avid sites that lack PSMA avidity ${ }^{(7)}$. Interestingly, the excluded group had poor outcome and shorter overall survival $^{(22)}$.

\section{Administration of ${ }^{177}$ lutetium- PSMA:}

PSMA-RNT should be given by well-trained nuclear medicine team. Before administration the patient is informed of the benefit and risks of this treatment as well as provided with radiation safety instruction. In Australia, oral hydration with $1.5 \mathrm{~L}$ of water is recommended on the day of therapy, intravenous hydration is an alternative if there is non-compliance with adequate oral hydration, ${ }^{177}$ Lu-PSMA-617 is administered by slow intravenous injection over $2-10 \mathrm{~min}$ (23). 
In Europe, some centers infuse 177Lu-PSMA617 at a slower rate and use routine intravenous fluid for hydration after ${ }^{(17)}$.

The patient stays under observation and then released after meeting the threshold of discharge according to national radiation protection regulations.

One time point post-therapy imaging at 24-48 hours, including whole body planar acquisition and SPECT/CT provides sufficient information regarding presence of targetable PSMA-expressing disease and can be used to monitor response in subsequent cycles. The optimal number of cycles required is still to be determined; it varies according to the response, toxicity, and disease burden. Studies showed variation with 2-6 cycles of 177Lu- PSMA RNT at a minimum 6 weeks interval between cycles and radioactivity in the range of $2-9.3 \mathrm{GBq}^{(6,7,14,16,17}$ and 24).

\section{Follow-up and response assessment:}

A combination of clinical, biochemical and radiographic assessment is required. Blood tests, including complete blood count and serum creatinine or clearance tests should be performed 2-4 weeks after treatment. Biochemical response assessment with tumor marker PSA should be tested in 4-week intervals, results of studies for mCRPC patients who were treated with $177 \mathrm{Lu}-\mathrm{PSMA}$
RNT and achieved $\geq 50 \%$ PSA decline were associated with significantly longer duration of response and longer overall survival compared to $<50 \%$ decline ${ }^{(\mathbf{8}, 25,26)}$.

MI assessment using PSMA PET/CT and FDG PET/CT provides information regarding disease heterogeneity and mixed responses, in addition to its superiority to CT, especially for evaluating bone metastases ${ }^{(23,27)}$.

Most mCRPC patients are subject to relapse, re-treatment with177Lu-PSMA RNT can be considered, particularly in patients with good initial response.

\section{Side effect:}

The salivary gland has been shown to receive the highest absorbed dose, causing mild to moderate xerostomia, different ideas have been adopted by some centers aiming to reduce this side effect, including local cooling

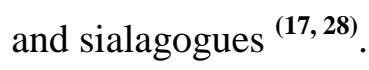

Hepatotoxicity is a common side effect. In $\mathrm{Lu}$ PSMA study post-treatment grade 3-4 anemia, thrombocytopenia, and neutropenia were found in $10 \%, 10 \%$, and $6 \%$ of the patients, respectively ${ }^{(7)}$.

In German multicenter study post-treatment grade 3-4 anemia, thrombocytopenia, and leukopenia, occurred at lower rate, 10\%, 4\%, and $3 \%$, respectively ${ }^{(\mathbf{1 7})}$. 
Renal toxicity is less common side effect. Yordanova et al, reported no grade 3-4 posttreatment nephrotoxicity on 55 patients (29). Hofman et al, reported $10 \%$ short term renal toxicity ${ }^{(7)}$.

Pain, nausea and vomiting have been reported. In TheraP trial, patients are

\section{REFERENCES:}

1. Bray F, Ferlay J, Soerjomataram I, et al. Global cancer statistics 2018: GLOBOCAN estimates of incidence and mortality worldwide for 36 cancers in 185 countries. CA: a cancer journal for clinicians. 68(6):394-424; 2018.

2. Parker C, Gillessen S, Heidenreich A, et al. Cancer of the prostate: ESMO Clinical Practice Guidelines for diagnosis, treatment and follow-up. Annals of oncology: official journal of the European Society for Medical Oncology. 26 Suppl 5:v69-77; 2015.

3. Gillessen S, Omlin A, Attard G, et al. Management of patients with advanced prostate cancer: recommendations of the $\mathrm{St}$ Gallen Advanced Prostate Cancer Consensus Conference (APCCC) 2015. Annals of oncology: official journal of the European Society for Medical Oncology. 30 (12):e3; 2019.

4. O'Keefe DS, Bacich DJ, Huang SS, et al. A Perspective on the Evolving Story of PSMA Biology, PSMA-Based Imaging, and prescribed a 3-day course of dexamethasone and antiemetic ${ }^{(20)}$.

\section{Future direction:}

Consideration of PSMA RNT at earlier stage of prostate cancer. Efficacy and safety of combining beta and alpha particle emitter as well as combing PSMA RNT with other treatment modalities.

Endoradiotherapeutic Strategies. Journal of nuclear medicine: official publication, Society of Nuclear Medicine. 59 (7):1007$13 ; 2018$.

5. Hofman MS, Lawrentschuk N, Francis $\boldsymbol{R} \boldsymbol{J}$, et al. Prostate-specific membrane antigen PET-CT in patients with high-risk prostate cancer before curative-intent surgery or radiotherapy (pro PSMA): a prospective, randomized, multicenter study. Lancet. 395(10231):1208-16; 2020.

6. Heck MM, Tauber R, Schwaiger S, et al. Treatment Outcome, Toxicity, and Predictive Factors for Radio ligand Therapy with (177)Lu-PSMA-I\&T in Metastatic Castration-resistant Prostate Cancer. European urology. 75(6):920-6; 2019.

7. Hofman MS, Violet J, Hicks RJ, et al. [(177)Lu]-PSMA-617 radionuclide treatment in patients with metastatic castrationresistant prostate cancer (LuPSMA trial): a single-center, single-arm, phase 2 study. The Lancet Oncology. 19(6):825-33; 2018. 
8. Violet J, Sandhu S, Iravani A, et al. Long term follow-up and outcomes of retreatment in an expanded 50 patient singlecenter phase II prospective trial of Lutetium-177 ((177)Lu) PSMA-617 theranostics in metastatic castrate-resistant prostate cancer. Journal of nuclear medicine: official publication, Society of Nuclear Medicine; 2019.

\section{Giesel FL, Hadaschik B, Cardinale J,} et al. F-18 labelled PSMA-1007: bio distribution, radiation dosimetry and histopathological validation of tumor lesions in prostate cancer patients. European journal of nuclear medicine and molecular imaging. 44(4):678-88; 2017.

10. Rowe SP, Gage KL, Faraj SF, et al. (1)(8)F-DCFBC PET/CT for PSMA-Based Detection and Characterization of Primary Prostate Cancer. Journal of nuclear medicine: official publication, Society of Nuclear Medicine. 56(7):1003-10; 2015.

11. 11. Rowe SP, Gorin MA, Pomper MG. Imaging of Prostate-Specific Membrane Antigen Using [(18) F] DCFPyL. PET clinics. 12(3):289-96; 2017.

12. Niaz MJ, Batra JS, Walsh RD, et al. Pilot Study of Hyper fractionated Dosing of Lutetium-177-Labeled AntiprostateSpecific Membrane Antigen Monoclonal Antibody J591 ((177) Lu-J591) for Metastatic Castration-Resistant Prostate Cancer. The oncologist. 2020.
13. Kratochwil $C$, Bruchertseifer $F$, Rathke H, et al. Targeted alpha-Therapy of Metastatic Castration-Resistant Prostate Cancer with (225)Ac-PSMA-617: Dosimetry Estimate and Empiric Dose Finding. Journal of nuclear medicine: official publication, Society of Nuclear Medicine. 58(10):1624-31; 2017.

14. Emmett L, Crumbaker M, Ho B, et al. Results of a Prospective Phase 2 Pilot Trial of (177)Lu-PSMA-617 Therapy for Metastatic Castration-Resistant Prostate Cancer Including Imaging Predictors of Treatment Response and Patterns of Progression. Clinical genitourinary cancer. 17(1):15-22; 2019.

15. McBean R, O'Kane B, Parsons $R$, et al. Lu177-PSMA therapy for men with advanced prostate cancer: Initial 18 months experience at a single Australian tertiary institution. Journal of medical imaging and radiation oncology. 63(4):538-45; 2019.

\section{Ahmadzadehfar H, Wegen S,} Yordanova A, et al. Overall survival and response pattern of castration-resistant metastatic prostate cancer to multiple cycles of radio ligand therapy using [(177)Lu]LuPSMA-617. European journal of nuclear medicine and molecular imaging. 44(9):1448-54; 2017. 
17. Rahbar K, Ahmadzadehfar $H$, Kratochwil $\boldsymbol{C}$, et al. German Multicenter Study Investigating 177Lu-PSMA-617 Radioligand Therapy in Advanced Prostate Cancer Patients. Journal of nuclear medicine: official publication, Society of Nuclear Medicine. 58(1):85-90; 2017.

18. Yadav MP, Ballal S, Bal C, et al. Efficacy and Safety of 177Lu-PSMA-617 Radio ligand Therapy in Metastatic Castration-Resistant Prostate Cancer Patients. Clinical nuclear medicine. 45(1):1931; 2020 .

19. Rahbar K, Bodei L, Morris MJ. Is the Vision of Radio ligand Therapy for Prostate Cancer Becoming a Reality? An Overview of the Phase III VISION Trial and Its Importance for the Future of Theranostics. JNM:. 60(11):1504-6; 2019.

20. Hofman MS, Emmett L, Violet J, et al. TheraP: a randomized phase 2 trial of (177) Lu-PSMA-617 theranostic treatment vs cabazitaxel in progressive metastatic castration-resistant prostate cancer (Clinical Trial Protocol ANZUP 1603). BJU international.124 Suppl 1:5-13; 2019.

21. Scher HI, Morris MJ, Stadler WM, et al. Trial Design and Objectives for Castration-Resistant Prostate Cancer: Updated Recommendations From the Prostate Cancer Clinical Trials Working Group 3. Journal of clinical oncology: official journal of the American Society of Clinical Oncology. 34(12):1402-18; 2016.
22. Thang SP, Violet J, Sandhu S, et al. Poor Outcomes for Patients with Metastatic Castration-resistant Prostate Cancer with Low Prostate-specific Membrane Antigen (PSMA) Expression Deemed Ineligible for (177)Lu-labelled PSMA Radio ligand Therapy. European urology oncology. 2(6):670-6; 2019.

23. Iravani A, Violet J, Azad A, et al. Lutetium-177 prostate-specific membrane antigen (PSMA) theranostics: practical nuances and intricacies. Prostate cancer and prostatic diseases. 23(1):38-52; 2020.

24. Brauer A, Grubert LS, Roll W, et al. (177) Lu-PSMA-617 radio ligand therapy and outcome in patients with metastasized castration-resistant prostate cancer. European journal of nuclear medicine and molecular imaging. 44(10):1663-70; 2017.

25. Rahbar K, Boegemann M, Yordanova $\boldsymbol{A}$, et al. PSMA targeted radio ligand therapy in metastatic castration resistant prostate cancer after chemotherapy, a biraterone and/or enzalutamide. A retrospective analysis of overall survival. European journal of nuclear medicine and molecular imaging. 45(1):12-9; 2018.

26. Ahmadzadehfar $\boldsymbol{H}$, Schlolaut $S$, Fimmers $R$, et al. Predictors of overall survival in metastatic castration-resistant prostate cancer patients receiving [(177)Lu]Lu-PSMA-617 radio ligand therapy. Oncotarget. 8(61):103108-16; 2017. 
27. Fech V, Alberts I, Rominger A, et al. PSMA-ligand PET allows a more accurate therapeutic response evaluation of bone metastases in prostate cancer compared to computed tomography. Nuklearmedizin Nuclear medicine; 2019.

28. Yilmaz B, Nisli S, Ergul N, et al. Effect of External Cooling on (177)LuPSMA Uptake by the Parotid Glands. Journal of nuclear medicine: official publication,
Society of Nuclear Medicine. 60(10):138893; 2019.

29. Yordanova A, Becker A, Eppard E, et al. The impact of repeated cycles of radio ligand therapy using [(177) Lu Lu-PSMA617 on renal function in patients with hormone refractory metastatic prostate cancer. European journal of nuclear medicine and molecular imaging. 44(9):1473-9; 2017. 